\title{
Subsurface, Thermoelastic Line Source Excitation of a Transversely Isotropic Half Space
}

James B Spicer*, Fan W. Zeng, Lauren R. Olasov

Department of Materials Science and Engineering

The Johns Hopkins University

3400 North Charles Street

Baltimore, MD 21218

\begin{abstract}
In this work, mathematical descriptions of material displacements in the wavefield surrounding a subsurface, thermoelastic line source in a transversely isotropic half space are given. These are derived from continuum representations that yield wave equations describing material motion that are solved using transform techniques. These solutions provide insight into the overall character of these sources in anisotropic materials and can also be used to model more generalized source distributions that might occur in a range of physical systems. Analytical, closed form solutions for epicentral displacements are derived and are graphically represented for particular source locations below the surface of a titanium half space. These results illustrate general aspects of the wavefield including the nature of various component modes of the displacement waveform. Wavefront characteristics associated with these modes are considered along with the nature of direct shear wave emission by this type of source. Mathematical results for displacements are used to illustrate how the surface-borne thermoelastic line source can be represented as a surface, shear stress dipole and are also used to represent more complicated subsurface source distributions that might occur in physical systems.
\end{abstract}

Keywords: transverse isotropy, thermoelastic, line source, elastic wave, wavefronts

\section{Introduction}

Subsurface line sources of elastic waves have been studied in fields from quantitative seismology [1] to medical imaging [2] in order to understand the character of the wavefields produced by these sources and to use the various wavefield components to characterize the nature (geometry and elastic properties) of the system being studied. In some cases, the line source of interest is primarily mechanical [3-5] while in others it has a thermoelastic nature being derived from a source of thermal energy that couples to elastic deformations in the system. The thermoelastic line source is the focus of this work. This type of source could be derived from a range of physical and/or chemical processes (e.g. endothermic/exothermic chemical reactions, phase transformations, photothermal processes) that could take place along a line. One of the most frequently studied is the laser line source in which light is focused to a line-like geometry in/on a material and acts as a thermoelastic source in regions where optical absorption occurs [6-12].

Corresponding author. e-mail: spicer@jhu.edu (James B. Spicer)

(C) 2016. This manuscript version is made available under the Elsevier user license

http://www.elsevier.com/open-access/userlicense/1.0/ 
Under conditions that produce subsurface absorption or that transport thermal energy away from absorption sites, subsurface sources will result and the associated elastic wavefield will depend on their distribution [13,14]. If these sources form a linear array below the surface, then a single line source representation could be sufficient for modeling purposes. More complicated distributions can be modeled using a suitable superposition of individual line sources.

Most studies that consider subsurface line sources assume isotropic properties for the host material [15]. This type of description can be useful for many materials, but most materials systems display some level of anisotropy owing to underlying crystallinity or as result of preferred orientational arrangement of constituent components and defects in the overall material structure. In many cases, when anisotropy is present, materials can be expected to display transverse isotropy. Materials systems that are transversely isotropic with respect to their thermal and elastic properties include those with hexagonal symmetry [16] as well as those that undergo particular processing that results in higher transverse symmetry. This anisotropy might be small and might not be taken into account when characterization is performed, but this does not mean it should be ignored especially since it might ultimately impact materials performance. There is clearly a need to develop models for interpretation of data gathered in transversely isotropic materials systems [17-19]. The purpose of the work presented here is to provide descriptions of material displacements in the wavefield surrounding a subsurface, thermoelastic line source in a transversely isotropic half space. These descriptions provide insight into the overall character of these sources in anisotropic materials and can also be used to model more generalized source distributions that might occur in a range of physical systems. Explicit expressions for displacements along the symmetry axis for a receiver in an epicentral location are derived and are computed to illustrate various general aspects of the wavefield. In particular, the characteristics of wavefronts associated with various modes are considered along with the nature of direct shear wave emission by this type of source. This type of emission does not occur in isotropic systems. Finally, the relationship between solutions obtained in this work to those associated with the application of boundary tractions/stresses is derived - this shows how the surface-borne thermoelastic line source can be represented as a surface, shear stress dipole $[19,20]$.

\section{Modeling the Thermoelastic Line Source}

The presentation largely follows work previously presented by Payton on line source excitation of transversely isotropic systems [16, 21-25] but the source specification in the current work differs significantly. Also, Payton considers a range of systems in which wavefield characteristics are quite complicated, but the focus of this presentation is only on systems that satisfy the requirements for Class I materials $[16,26,27]$. Aspects of the current work also relate closely to previous studies by Hurley $[18,20]$, but this treatment focuses principally on subsurface sources and the epicentral displacements 
produced by these types of sources and includes explicit, closed-form expressions for these displacements that have not been shown previously.

\subsection{Specification of the Source}

We start this development with the equation of motion for a general, anisotropic, thermoelastic solid:

$$
c_{i j k l} u_{l, k j}+f_{i}-\beta_{i j} T_{, j}=\rho u_{i, t t}
$$

where $c_{i j k l}$ is the elastic stiffness tensor, $u_{l}$ is the material displacement in the $l$ direction, $f_{i}$ is the body force acting in the $i$ direction, $\rho$ is the material density, $\beta_{i j}$ is the thermal stress coefficient tensor and $T$ is the temperature rise above the background, ambient level. Subscripting notation has been used where the indices range from 1 to 3 and represent spatial directions/variables while the index $t$ represents time. The summation convention has been used and subscripts after commas indicate differentiation with respect to the corresponding variable. Equation (1) is re-written as follows so that normalized stiffnesses can be used to describe the elastic response of the material [16]:

$$
c_{i j k l} v_{l, k j} / c_{2323}+\left(f_{i}-\beta_{i j} T_{, j}\right) / z_{2323}=\rho v_{i, \tau \tau}
$$

where $v_{i}=u_{i}\left(c_{2323} / \rho\right)^{1 / 2}, \quad z_{2323}=\left(\rho c_{2323}\right)^{1 / 2}$ and $\tau=\left(c_{2323} / \rho\right)^{1 / 2} t$. This normalization simplifies notation to be used in this development and introduces the reduced displacement, $v_{i}$, the reduced time, $\tau$ as well as the acoustic impedance, $z_{2323}$.

In the subsequent development, all body forces will be assumed to be zero, $f_{i}=0$, and all solutions will assume the thermal stress approximation - namely, coupling of elastic disturbances to thermal transport will be ignored. In addition, thermal diffusion will be suppressed by assuming that the thermal conductivity in the medium is zero. Under these conditions, a subsurface, line, heating source aligned along the $x_{1}$ axis with impulse time dependence (schematically shown in Fig. 1), can be described through the temperature field term in Eq. (1) as follows [19]:

$$
\beta_{i j} T_{, j}=\left(c_{i j k l} \alpha_{k l}\right)\left(\theta \delta\left(x_{2}\right) \delta\left(x_{3}\right) H(t)\right)_{, j}
$$

where $\alpha_{k l}$ is the linear thermal expansion coefficient tensor $\left(\beta_{i j}=c_{i j k l} \alpha_{k l}\right)$ and $\theta$ is heat source power per unit length divided by the heat capacity of the material. Evaluating the derivatives in Eq. (3) yields the following two "thermoelastic forces" that can be used with Eq. (2):

$$
\begin{aligned}
& \varphi_{2}=\beta_{22} T_{, 2}=\theta\left(c_{2222} \alpha_{22}+c_{2233} \alpha_{33}\right) \delta\left(x_{2}\right)_{, 2} \delta\left(x_{3}\right) H(t)=\phi_{2} \delta\left(x_{2}\right)_{, 2} \delta\left(x_{3}\right) H(t) \\
& \varphi_{3}=\beta_{33} T_{, 3}=\theta\left(c_{3322} \alpha_{22}+c_{3333} \alpha_{33}\right) \delta\left(x_{2}\right) \delta\left(x_{3}\right)_{, 3} H(t)=\phi_{3} \delta\left(x_{2}\right) \delta\left(x_{3}\right)_{, 3} H(t)
\end{aligned}
$$


where $\phi_{2}=\theta\left(c_{2222} \alpha_{22}+c_{2233} \alpha_{33}\right)$ and $\phi_{3}=\theta\left(c_{3322} \alpha_{22}+c_{3333} \alpha_{33}\right)$.

\subsection{Solution Method and Analytical Results}

Having specified the essential content of the physical problem, we can proceed to perform the necessary mathematical manipulations to obtain the material displacements that result from this type of source. Noting symmetries associated with the source, Eq. (2) yields the following:

$$
\begin{aligned}
& \left(\beta v_{2,22}+v_{2,33}-v_{2, \tau \tau}\right)+\kappa v_{3,23}=\varphi_{2} / z_{2323} \\
& \kappa v_{2,23}+\left(\alpha v_{3,33}+v_{3,22}-v_{3, \tau \tau}\right)+=\varphi_{3} / z_{2323}
\end{aligned}
$$

where $\alpha=c_{3333} / c_{2323}, \beta=c_{1111} / c_{2323}$, and $\kappa=1+c_{2233} / c_{2323}$. These equations need to be solved subject to the appropriate boundary conditions. In this work, we are interested in analytical, closed form solutions and the process to obtain these will employ transform techniques since these will convert differential equations to algebraic expressions that can be manipulated readily. The following definitions for the various forward transforms will be used in the subsequent development:

$$
\begin{aligned}
\bar{v}_{i} & =\int_{0}^{\infty} \int_{-\infty}^{\infty} \int_{-\infty}^{\infty} v_{i} \exp \left(-i k_{2} x_{2}-i k_{3} x_{3}-s \tau\right) d x_{2} d x_{3} d \tau \\
& =\int_{-\infty}^{\infty} \int_{-\infty}^{\infty} \hat{v}_{i} \exp \left(-i k_{2} x_{2}-i k_{3} x_{3}\right) d x_{2} d x_{3}=\int_{-\infty}^{\infty} \beta_{i} \exp \left(-i k_{3} x_{3}\right) d x_{3}
\end{aligned}
$$

which are paired with the corresponding inverse transforms:

$$
\begin{aligned}
v_{i} & =-i(2 \pi)^{-3} \int_{C} \int_{-\infty}^{\infty} \int_{-\infty}^{\infty} \bar{v}_{i} \exp \left(i k_{2} x_{2}+i k_{3} x_{3}+s \tau\right) d k_{2} d k_{3} d s \\
& =-i(2 \pi)^{-2} \int_{C}^{\infty} \int_{-\infty}^{\infty} B / \exp \left(i k_{2} x_{2}+s \tau\right) d k_{2} d s=-i(2 \pi)^{-1} \int_{C} \hat{v}_{i} \exp (s \tau) d s
\end{aligned}
$$

Applying the forward transform operations to Eqs. (6) and (7) yields the following:

$$
\bar{v}_{2}\left(\beta k_{2}^{2}+k_{3}^{2}+s^{2}\right)+\bar{v}_{3}\left(\kappa k_{2} k_{3}\right)=-\left(\phi_{2} / z_{2323}\right)\left(i k_{2} / s\right)
$$

and

$$
\bar{v}_{2}\left(\kappa k_{2} k_{3}\right)+\bar{v}_{3}\left(k_{2}^{2}+\alpha k_{3}^{2}+s^{2}\right)=-\left(\phi_{3} / z_{2323}\right)\left(i k_{3} / s\right) .
$$

These equations are readily solved simultaneously to yield the displacement transforms:

$$
\bar{v}_{2}=\left[\left(k_{2}^{2}+\alpha k_{3}^{2}+s^{2}\right) \Phi_{2}-\left(\kappa k_{2} k_{3}\right) \Phi_{3}\right] \div D
$$

and

$$
\bar{v}_{3}=\left[\left(\beta k_{2}^{2}+k_{3}^{2}+s^{2}\right) \Phi_{3}-\left(\kappa k_{2} k_{3}\right) \Phi_{2}\right] \div D
$$


where $\Phi_{2}=-\left(\phi_{2} / z_{2323}\right)\left(i k_{2} / s\right), \Phi_{3}=-\left(\phi_{3} / z_{2323}\right)\left(i k_{3} / s\right)$ and

$$
D=\alpha k_{3}^{4}+k_{3}^{2}\left[(\alpha+1) s^{2}+k_{2}^{2}\left(1+\alpha \beta-\kappa^{2}\right)\right]+\left(k_{2}^{2}+s^{2}\right)\left(\beta k_{2}^{2}+s^{2}\right) .
$$

All that remains is to impose boundary conditions and complete the various inverse transform operations. To begin, we will consider inversion with respect to the transform pair $\left(x_{3}, k_{3}\right)$ since this will allow assessment of the boundary conditions. Let $k_{3 n}^{*}$ be the $n^{\text {th }}$ of the 4 roots to the bi-quadratic equation when $D=0$. These roots are expressed as follows:

$$
\begin{aligned}
& k_{31}^{*}=-k_{32}^{*}=(2 \alpha)^{-1 / 2}\left\{-\left[(\alpha+1) s^{2}+\chi k_{2}^{2}\right]-\zeta^{1 / 2}\right\}^{1 / 2} \\
& k_{33}^{*}=-k_{34}^{*}=(2 \alpha)^{-1 / 2}\left\{-\left[(\alpha+1) s^{2}+\chi k_{2}^{2}\right]+\zeta^{1 / 2}\right\}^{1 / 2}
\end{aligned}
$$

where the second subscript indexes each root, $\chi=1+\alpha \beta-\kappa^{2}$ and $\zeta=\left[(\alpha+1) s^{2}+\chi k_{2}^{2}\right]^{2}-4 \alpha\left(k_{2}^{2}+s^{2}\right)\left(\beta k_{2}^{2}+s^{2}\right)$. At this point, let $k_{3 n}=i k_{3 n}^{*}$. This change essentially demands $k_{31}>0$ and $k_{33}>0$ for Class I materials (see Ref. [16] pp. 133-134 for related discussion) and will prove convenient later in the development. With this definition, the denominator, $D$, may be reexpressed to yield the following:

$$
D^{-1}=\alpha^{-1}\left[\left(k_{3}^{2}+k_{31}^{2}\right)^{-1}-\left(k_{3}^{2}+k_{33}^{2}\right)^{-1}\right]\left(k_{33}^{2}-k_{31}^{2}\right)^{-1} .
$$

Since $\left(k_{3}^{2}+k_{3 n}^{2}\right)^{-1}=\left(2 i k_{3}\right)^{-1}\left[\left(k_{3 n}-i k_{3}\right)^{-1}-\left(k_{3 n}+i k_{3}\right)^{-1}\right]$, the transforms in Eqs. (12) and (13) have simple poles at $k_{3}= \pm i k_{3 n}$. Inversion of $\bar{v}_{3}$ with respect to $\left(x_{3}, k_{3}\right)$ can be performed to get $\tilde{v}_{3}=8 / 39+8 / 9$ where $\tilde{v}_{31}$ is the portion of the doubly-transformed solution for $v_{3}$ associated with the $k_{31}$ root and similarly for $\tilde{v}_{33}$. These parts of $\tilde{v}_{3}$ can be written as follows:

$$
\tilde{v}_{31}=-(2 \alpha)^{1 / 2}\left(k_{33}^{2}-k_{31}^{2}\right)^{-1}\left(s z_{44}\right)^{-1}\left[\left(\beta k_{2}^{2}+s^{2}-k_{31}^{2}\right) \Phi_{3}-\kappa k_{2}^{2} \Phi_{2}\right]\left[e^{k_{31} x_{3}} H\left(-x_{3}\right)-e^{-k_{31} x_{3}} H\left(x_{3}\right)\right]
$$

and

$$
\tilde{v}_{33}=(2 \alpha)^{1 / 2}\left(k_{33}^{2}-k_{31}^{2}\right)^{-1}\left(s z_{44}\right)^{-1}\left[\left(\beta k_{2}^{2}+s^{2}-k_{33}^{2}\right) \Phi_{3}-\kappa k_{2}^{2} \Phi_{2}\right]\left[e^{k_{33} x_{3}} H\left(-x_{3}\right)-e^{-k_{33} x_{3}} H\left(x_{3}\right)\right]
$$

where $H\left(x_{3}\right)$ is the Heaviside step function acting along the $x_{3}$ axis. Inverting these expressions with respect to the transform pairs $\left(x_{2}, k_{2}\right)$ and $(s, \tau)$ yields the displacements in the $x_{3}$ direction that result from a thermoelastic line source in an infinite space. Corresponding solutions for displacements in the $x_{2}$ direction could be written as well, but since the current focus is on displacements in the $x_{3}$ direction, only the results in Eqs. (19) and (20) are needed to complete this development. 
To obtain a corresponding solution for a half-space with a free surface located at $x_{3}=-d$, the following boundary conditions based on the disappearance of surface tractions must be satisfied:

$$
\left.\sigma_{33}\right|_{x_{3}=-d}=0,\left.\sigma_{23}\right|_{x_{3}=-d}=0
$$

where

$$
\sigma_{33}=c_{3333} u_{3,3}^{g}+c_{3322} u_{2,2}^{g}, \sigma_{23}=c_{2323}\left(u_{2,3}^{g}+u_{3,2}^{g}\right) \text {. }
$$

Here, $u_{i}^{g}$ refers to the general solution of the governing differential equation which is composed of homogeneous and inhomogeneous (or particular) solutions $-u_{i}^{g}=u_{i}^{h}+u_{i}$ or $\tilde{v}_{3}^{g}=(\$ / 39+8 / 9)+(\$ / 99+8 / 9)$ for reduced displacements in the transform domain. The inhomogeneous solution corresponds to infinite space and has already been written so it can be used in the boundary conditions when they are expressed in a reduced form in the doubly transformed space:

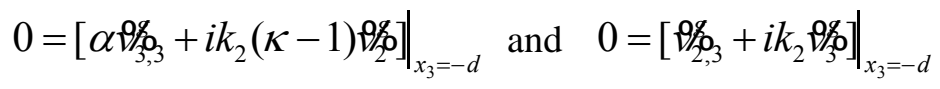

where $\tilde{v}_{i}^{g}=8 / \%+\beta / c$. Based on the form obtained for the inhomogeneous solution, the following homogeneous solutions are assumed:

$$
\tilde{v}_{2}^{h}=A_{1} e^{-k_{31} x_{3}}+A_{3} e^{-k_{33} x_{3}} \text { and } \tilde{v}_{3}^{h}=B_{1} e^{-k_{31} x_{3}}+B_{3} e^{-k_{33} x_{3}}
$$

where $A_{n}$ and $B_{n}$ are undetermined coefficients that are needed to satisfy the given boundary conditions. The additional requirements on the undetermined coefficients come from Eq. (7) when the right hand side is taken to be zero (yielding the homogeneous form of the equation):

$$
A_{n} / B_{n}=R_{n}=\left(\alpha k_{3 n}^{2}-k_{2}^{2}-s^{2}\right)\left(i \kappa k_{2} k_{3 n}\right)^{-1} .
$$

This expression requires that solutions associated with $k_{31}$ and $k_{33}$ be linearly independent. It follows

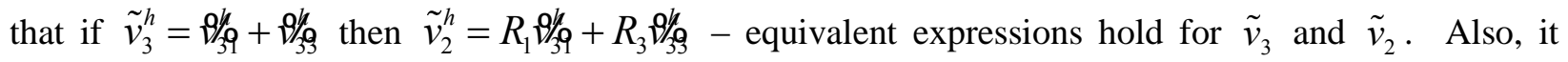
should be noted that if the sign of the propagation constant, $k_{3 n}$, changes then the sign of $R_{n}$ also changes. With this additional information in place, $\tilde{v}_{i}^{g}=\$ / \%+\$ / c$ can be used with the boundary conditions and values for $B_{1}$ and $B_{3}$ can be obtained. The algebra involved is tedious but yields the following results:

$$
\begin{aligned}
& B_{1}=\left[(a d+b c) \& /\left.9\right|_{x_{3}=0^{-}}+2 b d 8 /\left.9\right|_{x_{3}=0^{-}} e^{-\left(k_{31}+k_{33}\right) d}\right](a d-b c)^{-1} \\
& B_{3}=-\left[2 a c \% /\left.9\right|_{x_{3}=0^{-}} e^{-\left(k_{31}+k_{33}\right) d}+(a d+b c) 8 /\left.9\right|_{x_{3}=0^{-}} e^{-2 k_{33} d}\right](a d-b c)^{-1}
\end{aligned}
$$


with $a=k_{33}\left[\alpha k_{31}^{2}+(\kappa-1)\left(k_{2}^{2}+s^{2}\right)\right], b=k_{31}\left[\alpha k_{33}^{2}+(\kappa-1)\left(k_{2}^{2}+s^{2}\right)\right], c=\left[\alpha k_{31}^{2}+(\kappa-1) k_{2}^{2}-s^{2}\right]$ and $d=\left[\alpha k_{33}^{2}+(\kappa-1) k_{2}^{2}-s^{2}\right]$.

The complete solution, $\tilde{v}_{3}^{g}$, can be written as follows for regions of the half-space where $x_{3}>0$ :

$$
\begin{aligned}
\tilde{v}_{3}^{g}= & \left\{\left[-(a d-b c) \mathrm{Q} /\left.9\right|_{x_{3}=0^{-}}+(a d+b c) e^{-2 k_{31} d} \mathrm{Q} /\left.9\right|_{x_{3}=0^{-}}+2 b d e^{-\left(k_{31}+k_{33}\right) d} \mathrm{Q} /\left.3\right|_{x_{3}=0^{-}}\right] e^{-k_{31} x_{3}}\right. \\
& \left.+\left[-(a d-b c) \mathrm{Q} /\left.9\right|_{x_{3}=0^{-}}-(a d+b c) e^{-2 k_{33} d} \mathrm{Q} /\left.9\right|_{x_{3}=0^{-}}-2 a c e^{-\left(k_{31}+k_{33}\right) d} \mathrm{Q} /\left.9\right|_{x_{3}=0^{-}}\right] e^{-k_{33} x_{3}}\right\}(a d-b c)^{-1} .
\end{aligned}
$$

In this expression, terms of the form $\left.\tilde{v}_{3 i}\right|_{x_{3}=0^{-}}$should be evaluated using Eqns. (19) and (20) for negative values of $x_{3}$ in the limit as $x_{3} \rightarrow 0$. While various algebraic simplifications to this result can be made, this form for the solution will be used since it facilitates interpretations that will be made in subsequent sections. There are essentially six components of the solution given in Eq. (29) such that it can be

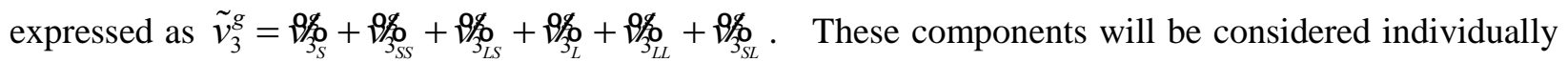
in the following to highlight the physical meaning behind each. Before doing so, it will be noted that significant simplification of the solution can be achieved by considering the factors $(a d+b c)$ and $(a d-b c)$ which appear in various terms. These quantities can be written as follows:

$$
a d+b c=\kappa\left(k_{33}+k_{31}\right)\left\{\left(k_{2}^{2}+s^{2}\right)\left[(2(\kappa-1)+\chi) k_{2}^{2}+\alpha s^{2}\right]-\alpha k_{31} k_{33} s^{2}\right\}
$$

and

$$
a d-b c=\kappa\left(k_{33}-k_{31}\right)\left\{\left(k_{2}^{2}+s^{2}\right)\left[(2(\kappa-1)+\chi) k_{2}^{2}+\alpha s^{2}\right]+\alpha k_{31} k_{33} s^{2}\right\} .
$$

The term $(a d-b c)$ corresponds to the usual Rayleigh denominator that appears in related treatments for isotropic materials [15].

The overall solution consists of six arrivals at the point of observation a distance of $x_{3}+d$ from the boundary - three associated with shear displacements and three associated with longitudinal displacements. If the receiver is epicentrally located at $x_{2}=0$, the six arrivals can be described as follows:

1. Direct Shear Wave. This portion of the wavefield travels a distance $x_{3}$ from the source to the receiver and is represented by $\tilde{v}_{3_{S}}^{g}=-8 /\left.3\right|_{x_{3}=0^{-}} e^{-k_{31} x_{3}}$. 
2. Reflected Shear-to-Shear Wave. This shear wave travels a distance of $x_{3}+2 d$ and includes reflection of a shear wave at the boundary. This arrival corresponds to $\tilde{v}_{3_{S S}}^{g}=(a d+b c) e^{-k_{31}\left(x_{3}+2 d\right)}$ \$/\% $\left.\right|_{x_{3}=0^{-}}(a d-b c)^{-1}$.

3. Longitudinal-to-Shear Conversion. This arrival is a shear wave that results from longitudinal wave conversion at the boundary (travels a distance $d$ as a longitudinal wave and a distance $x_{3}+d$ as a shear wave). This arrival is represented by $\tilde{v}_{3_{L S}}^{g}=2 b d e^{-k_{33} d-k_{31}\left(x_{3}+d\right)} \$ /\left.3\right|_{x_{3}=0^{-}}(a d-b c)^{-1}$.

4. Direct Longitudinal Wave. This portion of the wavefield corresponds to the longitudinal wave that travels a distance $x_{3}$ directly between the source and the receiver and is given by $\tilde{v}_{3_{L}}^{g}=-\vartheta /\left.9\right|_{x_{3}=0^{-}} e^{-k_{33} x_{3}}$.

5. Reflected Longitudinal-to-Longitudinal Wave. This is a longitudinal wave that travels a distance of $x_{3}+2 d$ and results from longitudinal wave reflection at the boundary. This arrival corresponds to $\tilde{v}_{3_{L L}}^{g}=-(a d+b c) e^{-k_{33}\left(x_{3}+2 d\right)} \mathrm{Q} /\left.9\right|_{x_{3}=0^{-}}(a d-b c)^{-1}$.

6. Shear-to-Longitudinal Conversion. This is a longitudinal wave that results from shear wave conversion at the boundary (travels a distance $d$ as a shear wave and a distance $x_{3}+d$ as a longitudinal wave). This arrival corresponds to $\tilde{v}_{3_{S L}}^{g}=-2 a c e^{-k_{31} d-\left(k_{33}+d\right) x_{3}} \$ /\left.9\right|_{x_{3}=0^{-}}(a d-b c)^{-1}$.

The solution given in Eq. (29) is in a doubly transformed space and inversions with respect to $k_{2}$ and $s$ need to be completed. The inverse transform with respect to $k_{2}$ is as follows:

$$
\widehat{v}_{3}^{g}=(2 \pi)^{-1} \int_{-\infty}^{\infty} \nabla \% \exp \left(i k_{2} x_{2}\right) d k_{2}=(\pi)^{-1} \operatorname{Re} \int_{0}^{\infty} \vartheta \% \exp \left(i k_{2} x_{2}\right) d k_{2}
$$

where $\hat{v}_{3}^{g}$ represents the Laplace transform of $v_{3}^{g}$ and the final equality in Eq. (32) results from $\tilde{v}_{3}^{g}$ being even with respect to $k_{2}$. The remainder of this treatment will be on epicentral displacements where $x_{2}=0$ since these are measured in various experiments. Also, the transforms simplify considerably when $x_{2}=0$ and yield analytical expressions for the displacements. Since $\tilde{v}_{3}^{g}$ in Eq. (32) depends on $k_{2}{ }^{2}$ - this suggests a change in the integration variable. Letting $\mathrm{K}_{2}=k_{2}{ }^{2}$ yields the following:

$$
\widehat{v}_{3}^{g}=(2 \pi)^{-1} \operatorname{Re} \int_{0}^{\infty} 8 \% \mathrm{~K}_{2}^{-1 / 2} d \mathrm{~K}_{2}
$$


While this change might not appear to have achieved any significant advantage, it allows the remaining inverse Laplace transform operation to be performed essentially by inspection.

For these final steps, we will consider the terms corresponding to each of the six arrivals in Eq. (29) separately as follows:

1. Direct Shear Wave. This wave corresponds to the term containing the exponential $e^{-k_{31} x_{3}}$ and will be referred to as $v_{3_{s}}^{g}$. For this term, let the variable $\tau^{\prime}=k_{31} x_{3} / s$ serve as the reduced time in a forward Laplace transform operation (see Eq. (9)). With this parameterization, $K_{2}$ can be interpreted to be a function of $\tau^{\prime}$ such that $d \mathrm{~K}_{2}=\frac{\partial \mathrm{K}_{2}}{\partial \tau^{\prime}} d \tau^{\prime}=\frac{s}{x_{3}} \frac{\partial \mathrm{K}_{2}}{\partial k_{31}} d \tau^{\prime}$. Combining Eq. (9) with (33) and (29) yields the following:

$$
v_{3_{S}}^{g}=-\left.i(2 \pi)^{-2} \int_{C} \operatorname{Re} \int_{0}^{\infty} H\left(\tau^{\prime}-x_{3}\right)\left(-\mathrm{K}_{2}^{-1 / 2} \frac{\partial \mathrm{K}_{2}}{\partial \tau^{\prime}} \vartheta / /\left.9\right|_{x_{3}=0^{-}}\right)\right|_{k_{31}=s \tau^{\prime} / x_{3}} \exp \left(-s \tau^{\prime}\right) \exp (s \tau) d \tau^{\prime} d s
$$

where

$$
\mathrm{K}_{2}=\left.(2 \beta)^{-1}\left\{\chi k_{3}^{2}-(1+\beta) s^{2}-\left[\left(\chi k_{3}^{2}-(1+\beta) s^{2}\right)^{2}-4 \beta\left(\alpha k_{3}^{2}-s^{2}\right)\left(k_{3}^{2}-s^{2}\right)\right]^{1 / 2}\right\}\right|_{k_{3}=k_{31}}
$$

and

$$
k_{33}=k_{31}^{-1}\left[\left(\mathrm{~K}_{2}+s^{2}\right)\left(\beta \mathrm{K}_{2}+s^{2}\right) / \alpha\right]^{1 / 2} .
$$

It should be noted that in arriving at Eq. (34), the change in the variable of integration from $\mathrm{K}_{2}$ to $\tau^{\prime}$ requires a change in the limits such that the lower limit $\mathrm{K}_{2}=0$ yields $\tau^{\prime}=x_{3}$. Introducing the Heaviside step function into the integrand allows the lower limit to be changed to zero. Also, the expression for $\mathrm{K}_{2}$ given in Eq. (35) can be obtained by solving the quadratic equation provided by Eq. (15) when $D=0$. Even though the derivative in the integrand can be obtained by differentiating Eq. (35) directly, an easier approach is to differentiate the quadratric equation for $\mathrm{K}_{2}$ (again, from Eq. (15) when $D=0$ ) and allowing the derivative to be expressed in terms of both $k_{3}$ and $\mathrm{K}_{2}$. This yields the result:

$$
\frac{\partial \mathrm{K}_{2}}{\partial k_{3}}=\frac{2 \chi k_{3} \mathrm{~K}_{2}+2(\alpha+1) s^{2} k_{3}-4 \alpha k_{3}^{3}}{2 \beta \mathrm{K}_{2}+(\beta+1) s^{2}-\chi k_{3}^{2}}
$$

Equation (34) appears to be a forward Laplace transform immediately followed by the inverse transform operation. The result for the displacement can be written immediately as follows:

$$
v_{3_{S}}^{g}=\left.(2 \pi)^{-1} H\left(\tau^{\prime}-x_{3}\right) \operatorname{Re}\left(-\mathrm{K}_{2}^{-1 / 2} \frac{\partial \mathrm{K}_{2}}{\partial \tau^{\prime}} \mathrm{Q} /\left.9\right|_{x_{3}=0^{-}}\right)\right|_{k_{31}=s \tau^{\prime} / x_{3}} .
$$


The displacement, $v_{3_{S}}^{g}$, can be expressed as a function of $\tau^{\prime}$ by noting that $k_{31}=s \tau^{\prime} / x_{3}$. Since $k_{3}=k_{31}$ in Eqs. (35)-(37), $\mathrm{K}_{2}, \frac{\partial \mathrm{K}_{2}}{\partial k_{3}}, k_{33}$ as well as $\left.\tilde{v}_{31}\right|_{x_{3}=0^{-}}$can be written directly and substituted into Eq. (38) to obtain $v_{3_{s}}^{g}$. The resulting equation is quite lengthy and it is not clear that expressing it would provide additional insight into the nature of the solution. It will not be shown here.

2. Reflected Shear-to-Shear Wave. This wave corresponds to the term containing the exponential $e^{-k_{31}\left(x_{3}+2 d\right)}$ and will be referred to as $v_{3_{S S}}^{g}$. For this term, let the variable $\tau^{\prime}=k_{31}\left(x_{3}+2 d\right) / s$ act as the reduced time in a forward Laplace transform operation. With this parameterization, $\mathrm{K}_{2}$ is a function of $\tau^{\prime}$ such that $d \mathrm{~K}_{2}=\frac{\partial \mathrm{K}_{2}}{\partial \tau^{\prime}} d \tau^{\prime}=\frac{s}{\left(x_{3}+2 d\right)} \frac{\partial \mathrm{K}_{2}}{\partial k_{31}} d \tau^{\prime}$. Combining Eq. (9) with (33) and (29) yields the following:

$v_{3_{S S}}^{g}=-\left.i(2 \pi)^{-2} \int_{C} \operatorname{Re} \int_{0}^{\infty} H\left(\tau^{\prime}-\left(x_{3}+2 d\right)\right)\left(\frac{a d+b c}{a d-b c} \mathrm{~K}_{2}^{-1 / 2} \frac{\partial \mathrm{K}_{2}}{\partial \tau^{\prime}} \vartheta /\left.39\right|_{x_{3}=0^{-}}\right)\right|_{k_{31}=s \tau^{\prime} /\left(x_{3}+2 d\right)} \exp \left(-s \tau^{\prime}\right) \exp (s \tau) d \tau^{\prime} d s$

where $\mathrm{K}_{2}, \frac{\partial \mathrm{K}_{2}}{\partial k_{3}}$ as well as $k_{33}$ have the same form as for the direct shear wave. As before, Eq. (39) appears to be a forward Laplace transform immediately followed by the inverse transform operation. The result can be written directly as follows:

$$
v_{3_{S S}}^{g}=\left.(2 \pi)^{-1} H\left(\tau^{\prime}-\left(x_{3}+2 d\right)\right) \operatorname{Re}\left(\frac{a d+b c}{a d-b c} \mathrm{~K}_{2}^{-1 / 2} \frac{\partial \mathrm{K}_{2}}{\partial \tau^{\prime}} \mathrm{Q} /\left.9\right|_{x_{3}=0^{-}}\right)\right|_{k_{31}=s \tau^{\prime} /\left(x_{3}+2 d\right)} .
$$

3. Longitudinal-to-Shear Conversion. This wave corresponds to the term containing the exponential $e^{-k_{33} d-k_{31}\left(x_{3}+d\right)}$ and will be referred to as $v_{3_{L S}}^{g}$. For this term, the variable $\tau^{\prime}=\left[k_{31}\left(x_{3}+d\right)+k_{33} d\right] / s$ acts as the reduced time. With this parameterization, $\mathrm{K}_{2}$ can once again be interpreted to be a function of $\tau^{\prime}$, but the relationship is more complicated. Combining Eq. (9) with (33) and (29) yields the following:

$$
v_{3_{L S}}^{g}=-\left.i(2 \pi)^{-2} \int_{C} \operatorname{Re} \int_{0}^{\infty} H\left(\tau^{\prime}-\left[\left(x_{3}+d\right)+x_{3} d / \alpha^{1 / 2}\right]\right)\left(2 b d \mathrm{~K}_{2}^{-1 / 2} \frac{\partial \mathrm{K}_{2}}{\partial \tau^{\prime}} 8 /\left.9\right|_{x_{3}=0^{-}}\right)\right|_{\tau^{\prime}=\left[k_{31}\left(x_{3}+d\right)+k_{33} d\right] / s} \exp \left(-s \tau^{\prime}\right) \exp (s \tau) d \tau^{\prime} d s
$$


In this case, evaluation of the derivative $d \mathrm{~K}_{2}=\frac{\partial \mathrm{K}_{2}}{\partial \tau^{\prime}} d \tau^{\prime}$ cannot be accomplished in the same way as was done in the previous two cases. Here we note that $\tau^{\prime}$ is a function of $\mathrm{K}_{2}$ such that

$$
\frac{\partial \mathrm{K}_{2}}{\partial \tau^{\prime}}=\left(\frac{\partial \tau^{\prime}}{\partial \mathrm{K}_{2}}\right)^{-1}=s\left(\left(x_{3}+d\right) \frac{\partial k_{31}}{\partial \mathrm{K}_{2}}+d \frac{\partial k_{33}}{\partial \mathrm{K}_{2}}\right)^{-1}
$$

The process for evaluating the various terms can be carried out by assuming values for $\mathrm{K}_{2}$, computing values for $k_{31}$ and $k_{33}$ with Eqs. (16) and (17) (along with subsequent definitions), and using these values to determine $\tau^{\prime}$ as well as the derivative terms $\frac{\partial k_{3 i}}{\partial \mathrm{K}_{2}}=\left.\left(\frac{\partial \mathrm{K}_{2}}{\partial k_{3}}\right)^{-1}\right|_{k_{3}=k_{3 i}}$. Having computed the values for these terms, the displacement is given by

$$
v_{3_{L S}}^{g}=\left.(2 \pi)^{-1} H\left(\tau^{\prime}-\left[\left(x_{3}+d\right)+x_{3} d / \alpha^{1 / 2}\right]\right) \operatorname{Re}\left(\frac{2 b d}{a d-b c} \mathrm{~K}_{2}^{-1 / 2} \frac{\partial \mathrm{K}_{2}}{\partial \tau^{\prime}} \vartheta /\left.9\right|_{x_{3}=0^{-}}\right)\right|_{\tau^{\prime}=\left[k_{31}\left(x_{3}+d\right)+k_{33} d\right] / s} .
$$

4. Direct Longitudinal Wave. This arrival corresponds to the term containing $e^{-k_{33} x_{3}}$ and will be referred to as $v_{3_{L}}^{g}$. A similar parameterization as was used for the direct shear term can be employed - the exponential suggesting the choice $\tau^{\prime}=k_{33} x_{3} / s$ such that $d \mathrm{~K}_{2}=\frac{\partial \mathrm{K}_{2}}{\partial \tau^{\prime}} d \tau^{\prime}=\frac{s}{x_{3}} \frac{\partial \mathrm{K}_{2}}{\partial k_{33}} d \tau^{\prime}$. Combining Eq. (9) with (33) and (29) yields the following:

$$
v_{3_{L}}^{g}=-\left.i(2 \pi)^{-2} \int_{C} \operatorname{Re} \int_{0}^{\infty} H\left(\tau^{\prime}-x_{3}\right)\left(-\mathrm{K}_{2}^{-1 / 2} \frac{\partial \mathrm{K}_{2}}{\partial \tau^{\prime}} 8 /\left.3\right|_{3_{3}=0^{-}}\right)\right|_{k_{33}=s \tau^{\prime} / x_{3}} \exp \left(-s \tau^{\prime}\right) \exp (s \tau) d \tau^{\prime} d s
$$

where

$$
\mathrm{K}_{2}=\left.(2 \beta)^{-1}\left\{\chi k_{3}^{2}-(1+\beta) s^{2}+\left[\left(\chi k_{3}^{2}-(1+\beta) s^{2}\right)^{2}-4 \beta\left(\alpha k_{3}^{2}-s^{2}\right)\left(k_{3}^{2}-s^{2}\right)\right]^{1 / 2}\right\}\right|_{k_{3}=k_{33}} .
$$

The derivative $\frac{\partial \mathrm{K}_{2}}{\partial k_{3}}$ as well as $k_{31}$ can be obtained using Eqs. (37) and (36) with $k_{3}=k_{33}$. Again, the result for this displacement can be written directly as follows:

$$
v_{3_{L}}^{g}=\left.(2 \pi)^{-1} H\left(\tau^{\prime}-x_{3} / \alpha^{1 / 2}\right) \operatorname{Re}\left(-\mathrm{K}_{2}^{-1 / 2} \frac{\partial \mathrm{K}_{2}}{\partial \tau^{\prime}} \mathrm{\vartheta} /\left.q_{3}\right|_{x_{3}=0^{-}}\right)\right|_{k_{33}=s \tau^{\prime} / x_{3}} .
$$

5. Reflected Longitudinal-to-Longitudinal Wave. This wave corresponds to the term containing the exponential $e^{-k_{33}\left(x_{3}+2 d\right)}$ and will be referred to as $v_{3_{L L}}^{g}$. For this term, let the variable $\tau^{\prime}=k_{33}\left(x_{3}+2 d\right) / s$ 
take on the role of reduced time in the Laplace transform operation in Eq. (8). The solution process follows the one outlined for other waves and yields the result:

$$
v_{3_{L L}}^{g}=\left.(2 \pi)^{-1} H\left(\tau^{\prime}-\left(x_{3}+2 d\right) / \alpha^{1 / 2}\right) \operatorname{Re}\left(-\frac{a d+b c}{a d-b c} \mathrm{~K}_{2}^{-1 / 2} \frac{\partial \mathrm{K}_{2}}{\partial \tau^{\prime}} 8 /\left.3\right|_{3_{3}=0^{-}}\right)\right|_{k_{33}=s \tau^{\prime} /\left(x_{3}+2 d\right)}
$$

where $\mathrm{K}_{2}$ and its derivative are the same as were used for the direct longitudinal wave.

6. Shear-to-Longitudinal Conversion. This wave corresponds to the term containing the exponential $e^{-k_{31} d-k_{33}\left(x_{3}+d\right)}$ and will be referred to as $v_{3_{S L}}^{g}$. For this term, let $\tau^{\prime}=\left[k_{31} d+k_{33}\left(x_{3}+d\right)\right] / s$. The rest of the development essentially follows that outlined for the longitudinal-to-shear conversion. Combining Eq. (9) with (33) and (29) yields the following:

$$
v_{3_{S L}}^{g}=-\left.i(2 \pi)^{-2} \int_{C} \operatorname{Re} \int_{0}^{\infty} H\left(\tau^{\prime}-\left[d+\left(x_{3}+d\right) / \alpha^{1 / 2}\right]\right)\left(-2 a c \mathrm{~K}_{2}^{-1 / 2} \frac{\partial \mathrm{K}_{2}}{\partial \tau^{\prime}} \vartheta / /\left.9\right|_{x_{3}=0^{-}}\right)\right|_{\tau^{\prime}=\left[k_{33} d+k_{31}\left(x_{3}+d\right)\right] / s} \exp \left(-s \tau^{\prime}\right) \exp (s \tau) d \tau^{\prime} d s
$$

such that the displacment is given by:

$$
v_{3_{S L}}^{g}=\left.(2 \pi)^{-1} H\left(\tau^{\prime}-\left[d+\left(x_{3}+d\right) / \alpha^{1 / 2}\right]\right) \operatorname{Re}\left(-2 a c \mathrm{~K}_{2}^{-1 / 2} \frac{\partial \mathrm{K}_{2}}{\partial \tau^{\prime}} \mathrm{Q} /\left.9\right|_{x_{3}=0^{-}}\right)\right|_{\tau^{\prime}=\left[k_{33} d+k_{31}\left(x_{3}+d\right)\right] / s} .
$$

The overall solution is simply the sum of the individual terms: $v_{3}^{g}=v_{3_{S}}^{g}+v_{3_{S S}}^{g}+v_{3_{L S}}^{g}+v_{3_{L}}^{g}+v_{3_{L L}}^{g}+v_{3_{S L}}^{g}$. Even though this expression for the displacements is complete and only requires evaluation of closedform terms, it is quite cumbersome and very little, if any, simplification can be accomplished unless special circumstances prevail. While various authors have explored elements of related solutions, few (if any) have considered all the terms individually especially those related to mode conversion at the boundary $[15,16]$.

\section{Computed Results - Graphical Presentation}

The overall character of the solution can be illustrated by computing displacement as a function of time for assumed conditions. Consider titanium for which $c_{1111}=162, c_{2233}=69, c_{3333}=181$ and $c_{2323}=46.7$ (all in units of GPa); $\alpha_{22}=9.23 \times 10^{-6}$ and $\alpha_{33}=9.57 \times 10^{-6}$ (both in units of $1 /{ }^{\circ} \mathrm{C}$ ). Suppose the point of observation is fixed such that the direct shear wave arrives at a time equal to $t=\tau\left(\rho / c_{2323}\right)^{1 / 2}$ with $\tau=1$ (the prime notation has been removed). Note that the units for $\tau$ correspond to length and are determined by the choice of units for time and the wavespeed, $\left(c_{2323} / \rho\right)^{1 / 2}$. Under these conditions, observation occurs at the location $x_{3}=\tau=1$. In addition, it will be assumed that the source is located at 
a distance $d=x_{3} / 20$ from the surface. For this geometry and material, the displacements at the point of observation as a function of reduced time will take the form shown in Fig. 2(a). In these results, the displacements have been divided by $\theta$ (the heat source power per unit length divided by the heat capacity) and multiplied by $\left(c_{2323} / \rho\right)^{1 / 2}$ (according to the original normalization carried out in Eq. (2)) and should be interpreted in terms of the related units. The most prominent features of the waveform are the first arrival (the direct longitudinal wave) at $\tau \approx 0.5$ and the longitudinal-to-longitudinal reflection - the negative-going peak after the first arrival. Since the waveform is composed of 6 different arrivals, it is useful to display each of these separately as is shown in Fig. 2(b). Here the first and second arrivals (L and LL respectively) are shown along with the mode-converted shear-to-longitudinal wave (SL), the direct shear wave (S) followed by the longitudinal-to-shear conversion (LS). The final arrival is the reflected shear wave (SS). Among the arrivals after the first two, the most significant is the longitudinalto-shear conversion - all others lack distinct onsets or have low amplitudes making them difficult to identify in the total displacement waveform. It should be noted that when computing the various contributions to the total displacement, arrivals that do not undergo mode conversion can be manipulated quite easily since reduced time is a direct input parameter. However, for mode-converted arrivals, reduced time is a derived quantity and, as a result, adding these terms to the others requires some care.

Additional results are shown in Fig. 3 where a series of displacement waveforms illustrates the effect of changing the depth of the source below the surface in titanium. For this series, the source-to-receiver distance is fixed so that the shear wave arrives at the reduced time $\tau=1$ (corresponding to $x_{3}=1$ ) while the source-to-surface distance $(d)$ takes on fractional values of $x_{3}$ - namely, $0.000,0.005,0.025,0.050$ and 0.075. This geometry fixes the arrival times for the direct longitudinal wave (at $\tau=\alpha^{-1 / 2} \approx 0.5$ ) and the direct shear wave ( $\tau=1.0$ ) - all other modes have arrival times that vary as the boundary is moved relative to the source with the most prominent being the longitudinal reflection. When the source is located far away from the surface, the reflected longitudinal wave is delayed relative to the direct wave by $\Delta \tau=2 d \alpha^{-1 / 2}$ and this difference decreases as the source approaches the surface. When the source is at the surface $(d=0.000)$, the wavefronts for the direct and reflected longitudinal waves cancel one another and the character of the overall waveform is changed significantly. The only distinct feature associated with shear displacements is the longitudinal-to-shear conversion. For the surface source, the arrival time for this component is $\tau=1.0$, but as the source moves away from the boundary, the arrival is delayed by $\Delta \tau=d\left(\alpha^{-1 / 2}+1\right) \quad$ - unlike for the reflected longitudinal wave, this shift is difficult to identify in the waveform owing to the nature of the shear wavefronts.

\section{Results for Special Conditions}




\subsection{Wavefront Arrivals}

The wavefront arrivals provide insight into the overall character of the waveforms produced by this type of source. Since the directly transmitted shear and longitudinal waves factor into all components of the wavefield, attention will be focused on these. For times approaching the arrival of the direct longitudinal wave, the following result is obtained for the displacement:

$$
\left.v_{3_{L}}^{g}\right|_{\tau \rightarrow\left(x_{3} / \alpha^{1 / 2}\right)^{+}}=(2 \pi)^{-1} H\left(\tau-x_{3} / \alpha^{1 / 2}\right)\left[\phi_{3} / z_{44}\right]\left[2 \alpha^{3 / 2}(\alpha-1) /\left(\alpha+\kappa^{2}-1\right)\right]^{1 / 2}\left(\tau-x_{3} / \alpha^{1 / 2}\right)^{-1 / 2} x_{3}^{-1 / 2} .(50
$$

The temporal dependence of the wavefront is a reciprocal, square root singularity - this is typical for line sources and indicates that the computed waveforms shown in this work are approximations to the actual result. In addition, the amplitude varies as the reciprocal, square root of the distance between the source and the point of observation - again, this is the usual result for line sources and is related to conservation of energy. When the reduced time approaches the arrival time for the direct shear wave, the following result is obtained for the epicentral displacement due to the shear wave:

$$
\left.v_{3_{S}}^{g}\right|_{\tau \rightarrow x_{3}}=(2 \pi)^{-1} H\left(\tau-x_{3}\right)\left[\kappa\left(\phi_{2}-\kappa \phi_{3} /(\alpha-1)\right) / z_{44}\right]\left[2 \alpha /\left(\beta+\kappa^{2}-\alpha \beta\right)\right]^{3 / 2}[2(\alpha-1)]^{1 / 2}\left(\tau-x_{3}\right)^{1 / 2} x_{3}^{-3 / 2}
$$

In this case, the amplitude varies with the square root of time and does not exhibit any singularity - this prevents this portion of the wavefield from contributing significantly to the overall epicentral

displacement. In addition, the wavefront displacement amplitude decreases more rapidly than $x_{3}^{-1 / 2}$ and does not contribute to overall energy transport to large distances $\left(x_{3}>>1\right)$. These results indicate that the directly transmitted longitudinal wave as well as displacements directly associated with this wave will dominate the overall character of epicentral displacements.

\subsection{Shear Wave Emission}

Direct emission of shear waves by the source is related to the material anisotropy and accounts for three of the terms in the full solution. Both elastic and thermal expansion anisotropy contribute to the existence of these portions of the wavefield for the geometry considered in this work. Shear waves are not directly generated in isotropic materials but do arise as a result of mode conversion at the boundary. This can be shown by considering the form of the source term as well as the solution for shear displacements in the unbounded medium (corresponding to $v_{31}$ or $\tilde{v}_{31}$ ). The thermoelastic source has components including terms introduced after Eqs. (4) and (5):

$$
\phi_{2}=\theta\left(c_{2222} \alpha_{22}+c_{2233} \alpha_{33}\right) \text { and } \phi_{3}=\theta\left(c_{3322} \alpha_{22}+c_{3333} \alpha_{33}\right) \text {. }
$$

For isotropic materials, all components of the thermal expansion tensor are equal $\left(\alpha_{22}=\alpha_{33}\right)$ and the stiffness tensor reduces to two independent components from the five for transversely isotropic materials. First, $c_{2222}=c_{3333}\left(\right.$ or $\alpha=\beta$ ) so that $\phi_{2}=\phi_{3}$ - this simplifies the form of the source and the thermally- 
generated stresses are isotropic under these conditions. Secondly, the symmetry simplifies stiffnessrelated terms used to express $\tilde{v}_{31}$ including $c_{2323}=c_{1212}$ and $c_{2233}=c_{2222}-2 c_{1212}$ so that $\kappa=\alpha-1$ and $\chi=2 \alpha$. With these equalities in place, according to Eq. (19), $\tilde{v}_{31}=0-$ no transverse displacements are emitted by the source. This immediately eliminates three of the six components of the wavefield and the remaining terms are wholly related to longitudinal wave emission by the source.

\subsection{Surface Expansion Line Source}

One interesting limit is the case when $d \rightarrow 0$ which yields the displacement field for a surface source. Returning to the transform of the general solution given in Eq. (29) and letting $d=0$ results in the following:

$$
\left.\tilde{v}_{3}^{g}\right|_{d=0}=-\left(\alpha s z_{44}\right)^{-1} \kappa k_{2}^{2}\left[\phi_{3}(1-\kappa)+\phi_{2} \alpha\right]\left[b e^{-k_{31} x_{3}}-a e^{-k_{33} x_{3}}\right](a d-b c)^{-1} .
$$

Having obtained this solution for the displacements in the $x_{3}$ direction from a thermoelastic, surface line source, an alternative specification can be created by assessing the equivalent boundary stresses that give rise to these same displacements. Using Eq. (53) along with the associated displacements in the $x_{2}$

direction $\left(\left.\tilde{v}_{2}^{g}\right|_{d=0}=\left.R_{1} \otimes \%\right|_{3=0}+R_{3} \otimes /\left.\%\right|_{d=0}-\right.$ see Eq. (25)), the stresses can be written for the doubly transformed domain as follows:

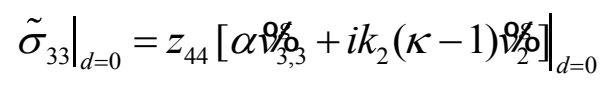

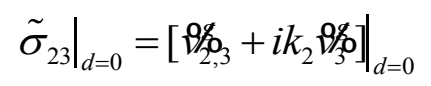

and evaluated at $x_{3}=0$ to yield:

$$
\left.\tilde{\sigma}_{33}\right|_{x_{3}=0} ^{d=0}=z_{44}\left(\kappa k_{31} k_{33}\right)^{-1}\left[-a \text { 裙| }\left.\right|_{\substack{d=0 \\ x_{3}=0}}-b \text { 腾 }\left.\right|_{\substack{d=0 \\ x_{3}=0}}\right]=0
$$

and

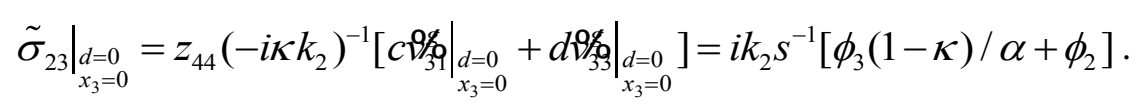

These expressions can be inverted with respect to the remaining transform variables to arrive at the following equivalent boundary conditions:

$$
\left.\sigma_{33}\right|_{x_{3}=0}=0 \quad \text { and }\left.\quad \sigma_{23}\right|_{x_{3}=0}=\left[\phi_{3}(1-\kappa) / \alpha+\phi_{2}\right] \delta\left(x_{2}\right)_{, 2} H(t) .
$$

This result was derived previously by Hurley using the method of images [20]. By explicitly deriving solutions for the buried thermoelastic source using a general approach, the surface source is simply a special case that can be modeled more directly using equivalent boundary conditions.

\subsection{Distribution of Subsurface Sources}


Another interesting source specification that contrasts to the single source on the half-space surface is a distribution of subsurface sources that are all contained in the plane defined by the line direction and the surface normal - see Fig. 4 for a schematic of the source configuration. This type of distributed source might occur in a variety of physical situations including those produced by subsurface photothermal conversion. If a total of $N$ discrete sources are arrayed according to a distribution where the source amplitude as a function of the depth below the surface for the $k^{\text {th }}$ source is $f\left(d_{k}\right)$, then the total displacement, $v_{3_{\text {Total }}}^{g}\left(x_{3}\right)$, resulting from the superposition of these can be expressed as follows:

$$
v_{3_{\text {Total }}}^{g}\left(x_{3}\right)=\sum_{k=0}^{N} f\left(d_{k}\right) v_{3}^{g}\left(x_{3}-d_{k}\right) .
$$

If these sources are arrayed in a regular fashion such that they are spaced at equal intervals below the surface (beginning at the surface), then $d_{k}=k \Delta d=k\left(d_{k+1}-d_{k}\right)$ and the total displacement can be computed according to Eq. (59). However, if $f\left(d_{k}\right)$ represents a continuous distribution function that is sampled at various locations, then the total displacement might be represented as follows:

$$
v_{3_{\text {Total }}}^{g}\left(x_{3}\right)=\left[\sum_{k=0}^{N} f\left(d_{k}\right) v_{3}^{g}\left(x_{3}-d_{k}\right) \Delta d\right]\left[\sum_{k=0}^{N} f\left(d_{k}\right) \Delta d\right]^{-1}
$$

where the denominator simply normalizes the computed displacement. The other factors do not change the result but are important if the distribution is exhaustively sampled - namely, as $\Delta d \rightarrow 0$ the following result emerges:

$$
v_{3_{\text {Total }}}^{g}\left(x_{3}\right)=\left[\int_{0}^{\infty} f(y) v_{3}^{g}\left(x_{3}-y\right) d y\right]\left[\int_{0}^{\infty} f(y) d y\right]^{-1}
$$

where the dummy variable, $y$, has been used to represent the source depth, $d$. Equation (61) is simply a convolution of the source distribution function with the displacement for the single line source normalized by the integrated source strength. Note that the original solution for the single line source located a distance $d$ from the surface is recovered from Eq. (61) if the distribution is a Dirac delta function where $f(y)=\delta(y-d)$.

\section{Discussion}

The intent of this work has been to derive the elastic displacements that occur in a transversely isotropic half-space resulting from the action of a subsurface, thermoelastic line source perpendicular to the symmetry axis and parallel to the surface. Analytical, closed form solutions for these displacements have been derived for receiver locations directly below the line source - namely, those that are epicentrally located - and computed waveforms have been shown for titanium. While the various results presented in this work are instructive, their value is primarily in the application to specific problems that might arise in 
physical systems that display transverse isotropy but do not directly adhere to restrictions that have been placed on the results shown in this work. Some additional comments regarding the application of results in this work are required. First, the assumption that the receiver is buried in a half-space below the source is not typically encountered. Receivers are generally located on surfaces. To generate a source-receiver geometry similar to the one assumed in this work, the receiver would likely reside on the back surface of a plate structure and boundary conditions on this second surface would need to be included in the development [28-30]. The displacements under these conditions can be developed from the results presented here by simply multiplying the various arrivals by the appropriate reflection coefficients - these are directly related to those already presented for reflection of the directly-emitted waves at the free surface. This treatment is consistent with ray descriptions of propagation in plates. Based on related treatments in isotropic materials, minor changes to the overall waveform shape are expected for reception on the back surface of a plate when compared to the buried receiver [18].

The next issue relates to the computation of wavefields associated with distributed sources. While the line source permits the development of simple solutions, many physical circumstances give rise to distributed sources and methods for computation of the resulting displacements should be considered. The convolution integral given in Eq. (61) provides a formal solution for one type of distributed source, but it is unknown whether or not there exist continuous source distributions giving rise to simple solutions that do not require numerical evaluation. Numerical integration is likely required to obtain the displacements from most sources, and the question naturally arises regarding the best computational approach to use. The convolution in Eq. (61) is one - this requires numerical evaluation of a single integral. However, the convolution could be carried out in the transform domain (before inversions were completed) and this could result in numerical evaluation of two, nested integrals. Indeed, under these conditions, a wider range of circumstances could be modeled since the temporal as well as the lateral spatial characteristics of the source might be incorporated into these integrals [9]. However, care must be taken during the associated numerical integrations - it might be more computationally efficient to simply solve the governing differential equations numerically as has been done by others previously [29]. While this approach does not take advantage of some elegant mathematics [31], it does utilize the capabilities of contemporary computational techniques and could reveal behaviors that are not readily identified when analytical techniques are employed. Essentially, a wide range of circumstances involving changes to the source geometry and temporal profile might be modeled quite rapidly once solution algorithms are in place.

Finally, restrictions regarding the overall solution as it relates to the essential system geometry should be considered. The case developed in this work has the free surface as well as the source perpendicular to 
the symmetry axis. However, since the source is linear, dependence of displacements on one of the three spatial coordinates is suppressed $-x_{1}$ for the case here. The source geometry renders the overall problem one of two spatial dimensions and this also occurs when the line is oriented along principal axes in other directions. Solutions for these other source orientations can be derived directly from the results developed in this work. If the surface as well as the source are parallel to the symmetry axis, then the overall system behaves isotropically - appropriate changes to subscripts as well as substitutions for the various moduli can be made to generate the corresponding solution. The final expression for epicentral displacements is more compact than the one presented here since three terms directly associated with shear wave emission disappear and remaining terms simplify considerably. The final source geometry of interest occurs when the surface is parallel to the symmetry axis and the source is perpendicular to it. Under these conditions, the solution procedure shown here can be used in its entirety with a simple exchange of subscripts ( $2 \ddot{A} 3$ ) along with a re-definition of the normalized moduli. Under these conditions, the longitudinal wavespeed changes while the shear wave travels at the same speed as for the case considered in the original development.

\section{Conclusions}

Mathematical expressions for the material displacements resulting from a subsurface, thermoelastic line source in a transversely isotropic half space have been presented. These expressions showed that six discrete wave arrivals can be expected in the wavefield. In particular, closed-form, analytical solutions for displacements associated with the six arrivals were derived for a receiver located directly below the source (in an epicentral location). Computed displacements in titanium showed large amplitude arrivals corresponding to longitudinal waves (directly transmitted and reflected from the surface) along with a smaller amplitude arrival associated with a shear wave produced by longitudinal wave conversion at the boundary. The directly transmitted longitudinal wavefront has a reciprocal, square root dependence on time as well as a reciprocal, square root dependence on the source-to-receiver distance - the temporal singularity at the longitudinal wavefront accounts for the large amplitudes in computed results. Shear waves have relatively small amplitudes for epicentral reception. In particular, the directly transmitted shear wave was shown to have a square root dependence on time yielding no singularity at the wavefront. The origin of this shear wave is directly related to the anisotropy of the system - it does not exist in isotropic systems. Computed results for titanium tend to indicate that the epicentral wavefront singularities for the direct and reflected longitudinal waves cancel one another in the limit as the source approaches the boundary leaving only higher order terms. This conclusion is supported by results for surface stresses that occur in the limit as the line moves to the boundary. In this limit, the source assumes the form of a surface, shear stress dipole with no contributions from the normal stress. Expressions have 
been developed for distributed sources that can be composed using superposition of subsurface line sources and it has also been shown that under appropriate conditions these expressions take on the form of a convolution between the source distribution function and the single line source solution developed in this work.

\section{Acknowledgements}

The authors gratefully acknowledge the support of the U.S. Department of Energy (DOE) through the Nuclear Energy University Program (NEUP) Contract No. 00118687.

\section{References}

[1] K Aki, PG Richards, Quantitative Seismology : Theory and Methods, W. H. Freeman, San Francisco, 1980.

[2] T Royston, Y Yazicioglu, F Loth. Surface response of a viscoelastic medium to subsurface acoustic sources with application to medical diagnosis, J.Acoust.Soc.Am. 113 (2003) 1109-1121.

[3] H Lamb. On the propagation of tremors over the surface of an elastic solid, Philosophical Transactions of the Royal Society of London Series a-Containing Papers of a Mathematical or Physical Character. 203 (1904) 1-42.

[4] SK Bhandari, R Chander. On the exact evaluation of displacements within a semi-infinite solid due to a buried line source, Proceedings of the Indian Academy of Sciences-Earth and Planetary Sciences. 93 (1984) 37-45.

[5] E Kausel. Lamb's problem at its simplest, Proceedings of the Royal Society A-Mathematical Physical and Engineering Sciences. 469 (2013).

[6] DA Hutchins, RJ Dewhurst, SB Palmer. Directivity patterns of laser-generated ultrasound in aluminum, J.Acoust.Soc.Am. 70 (1981) 1362-1369.

[7] JR Bernstein, JB Spicer. Line source representation for laser-generated ultrasound in aluminum, J.Acoust.Soc.Am. 107 (2000) 1352-1357.

[8] D Royer. Mixed matrix formulation for the analysis of laser-generated acoustic waves by a thermoelastic line source, Ultrasonics. 39 (2001) 345-354.

[9] I Arias, JD Achenbach. Thermoelastic generation of ultrasound by line-focused laser irradiation, Int.J.Solids Structures. 40 (2003) 6917-6935.

[10] JD Achenbach. Laser excitation of surface wave motion, J.Mech.Phys.Solids. 51 (2003) 18851902.

[11] WX Hu, ML Qian. Acoustic field excited by a pulsed laser line source in a cylinder, Ultrasonics. 44 (2006) E1187-E1190. 
[12] A Aharoni, K Jassby, M Tur. The Thermoelastic Surface Strip Source for Laser-Generated Ultrasound, J.Acoust.Soc.Am. 92 (1992) 3249-3258.

[13] K Telschow, R Conant. Optical and Thermal Parameter Effects on Laser-Generated Ultrasound, J.Acoust.Soc.Am. 88 (1990) 1494-1502.

[14] Y Guo, D Yang, Y Chang, W Gao. Effect of oblique force source induced by laser ablation on ultrasonic generation, Optics Express. 22 (2014) 166-176.

[15] KF Graff. Wave Motion in Elastic Solids, Dover Publications, New York, 1991.

[16] RG Payton. Elastic Wave Propagation in Transversely Isotropic Media, Martinus Nijhoff, The Hague, Netherlands, 1983.

[17] RL Ryan. Pulse propagation in a transversely isotropic half-space, J.Sound Vibrat. 14 (1971) 511$\&$.

[18] DH Hurley. An Investigation of the Anisotropic and Heterogeneous Nature of Laser Generated Ultrasound in Carbon-Fiber-Reinforced Epoxy and Single Crystal Materials, The Johns Hopkins University, Baltimore, Maryland, 1997.

[19] DH Hurley, JB Spicer. Line source representation for laser-generated ultrasound in an elastic transversely isotropic half-space, J.Acoust.Soc.Am. 116 (2004) 2914-2922.

[20] DH Hurley. Laser-generated thermoelastic acoustic: sources in anisotropic materials, J.Acoust.Soc.Am. 115 (2004) 2054-2058.

[21] RG Payton. 2-dimensional anisotropic elastic waves emanating from a point source, Proceedings of the Cambridge Philosophical Society-Mathematical and Physical Sciences. 70 (1971) 191-\&.

[22] RG Payton. Epicenter and epicentral-axis motion of a transversely isotropic elastic half-space, SIAM J Appl Math. 40 (1981) 373-389.

[23] RG Payton. Symmetry-axis elastic-waves for transversely isotropic media, Q Appl Math. 35 (1977) 63-73.

[24] RG Payton. Plane strain displacement and stress waves induced in a transversely isotropic elastic solid by a line source, Zeitschrift Fur Angewandte Mathematik Und Physik. 29 (1978) 262-272.

[25] EA Kraut. Advances in the theory of anisotropic elastic wave propagation, Rev.Geophys. 1 (1963) 401-448.

[26] MJP Musgrave, RG Payton. Criteria for elastic-waves in anisotropic media - a consolidation, Journal of Elasticity. 14 (1984) 269-285.

[27] RG Payton. Epicenter motion of a transversely isotropic elastic half-space due to a suddenly applied buried point source, Int.J.Eng.Sci. 17 (1979) 879-887. 
[28] B Xu, J Feng, G Xu, J Wang, H Sun, G Cao. Laser-generated thermoelastic acoustic sources and Lamb waves in anisotropic plates, Applied Physics A-Materials Science \& Processing. 91 (2008) 173-179.

[29] JJ Wang, BQ Xu, ZH Shen, XW Ni, J Lu. Numerical simulation of thermoelastic stress field and laser ultrasound in transversely isotropic plate, Japanese Journal of Applied Physics. 47 (2008) 956963.

[30] RL Weaver, W Sachse, KY Kim. Transient elastic waves in a transversely isotropic plate, Journal of Applied Mechanics-Transactions of the ASME. 63 (1996) 337-346.

[31] A Rahman, F Ahmad. Representation of the displacement in terms of scalar functions for use in transversely isotropic materials, J.Acoust.Soc.Am. 104 (1998) 3675-3676. 


\section{Figure Captions:}

Figure 1: Schematic illustration of the line source configuration modeled in this work. The source is perpendicular to the symmetry axis and parallel to the surface in a transversely isotropic material.

Figure 2: (a) Epicentral displacements for a source located a distance $d=0.05$ below the surface and a receiver located at $x_{3}=1$. The elastic constants and thermal expansion coefficient for titanium have been used. (b) The six components of the waveform shown in (a) where $\mathrm{L}$ is the direct longitudinal wave, LL is the reflected longitudinal wave, SL is the mode-converted shear-to-longitudinal wave, S is the direct shear wave, LS is the mode-converted longitudinal-to-shear wave and SS is the reflected shear wave.

Figure 3: Epicentral displacements in titanium for a receiver located at $x_{3}=1$ and a source located at the subsurface locations indicated next to each waveform. The direct and reflected longitudinal waves dominate the character of these waveforms when the source is subsurface $(d \neq 0)$.

Figure 4: Schematic illustration of source distributions that can be modeled using results presented in this work. Shading of sources indicates relative strengths. For collections of individual line sources as is shown for Cases 1-3, simple addition of waveforms can be used. In the limit of a continuous distribution as is shown in Case 4, convolution is required. 
Figure 1

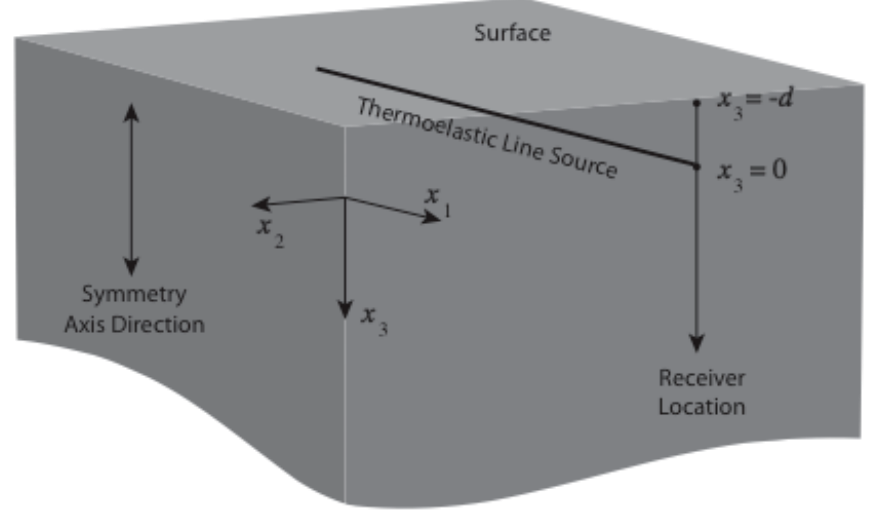


Figure 2
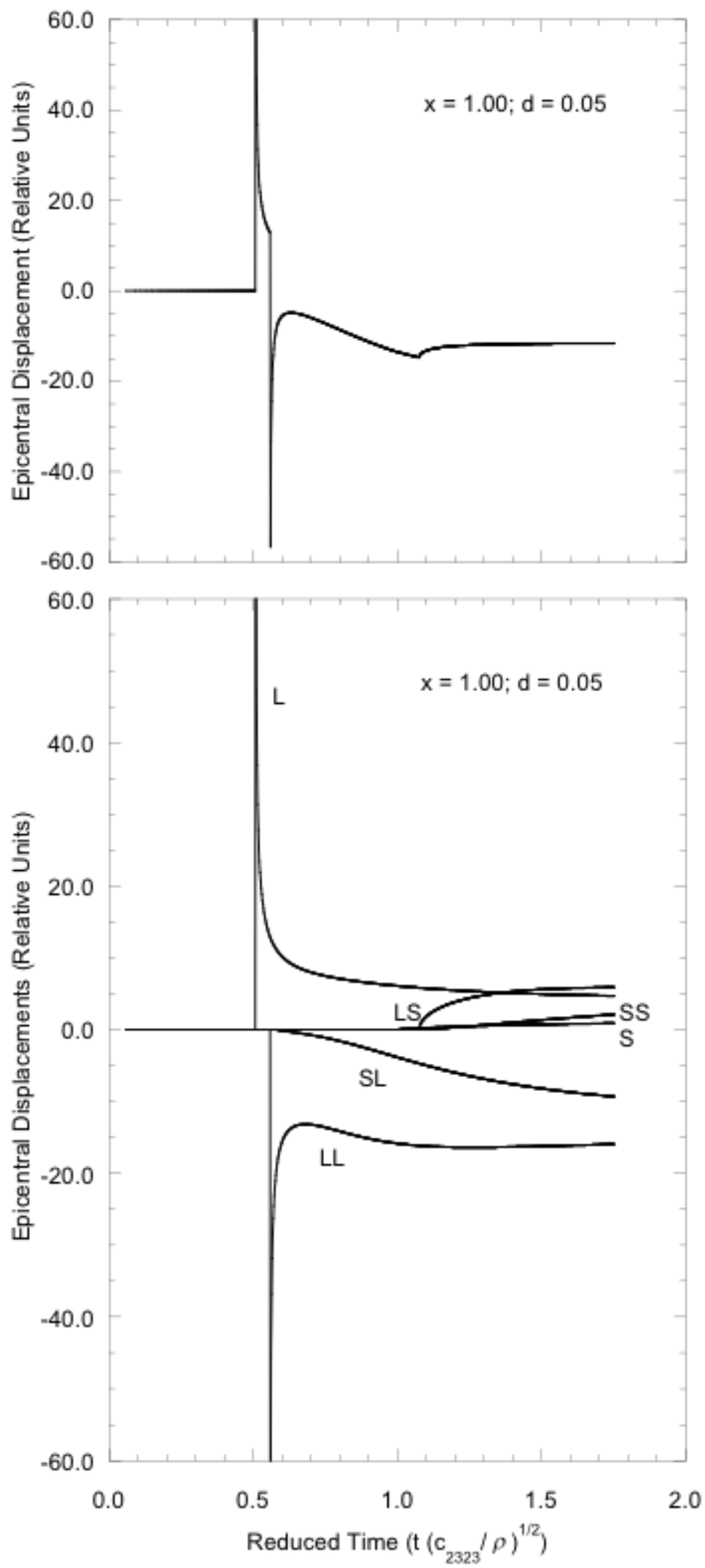
Figure 3
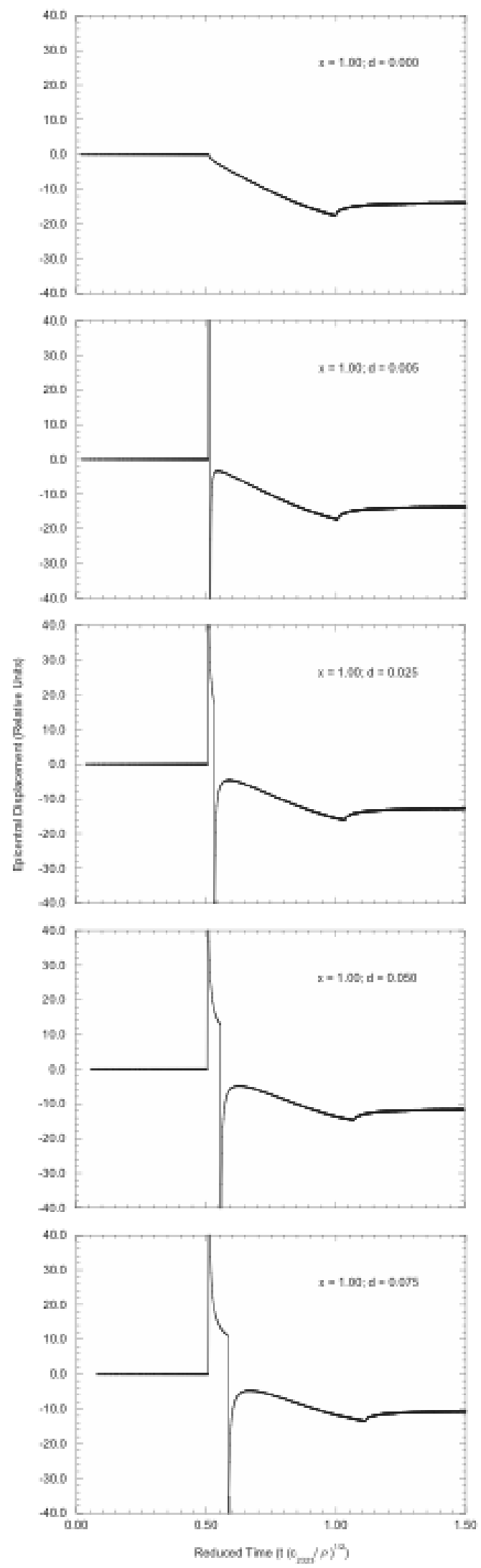
Figure 4

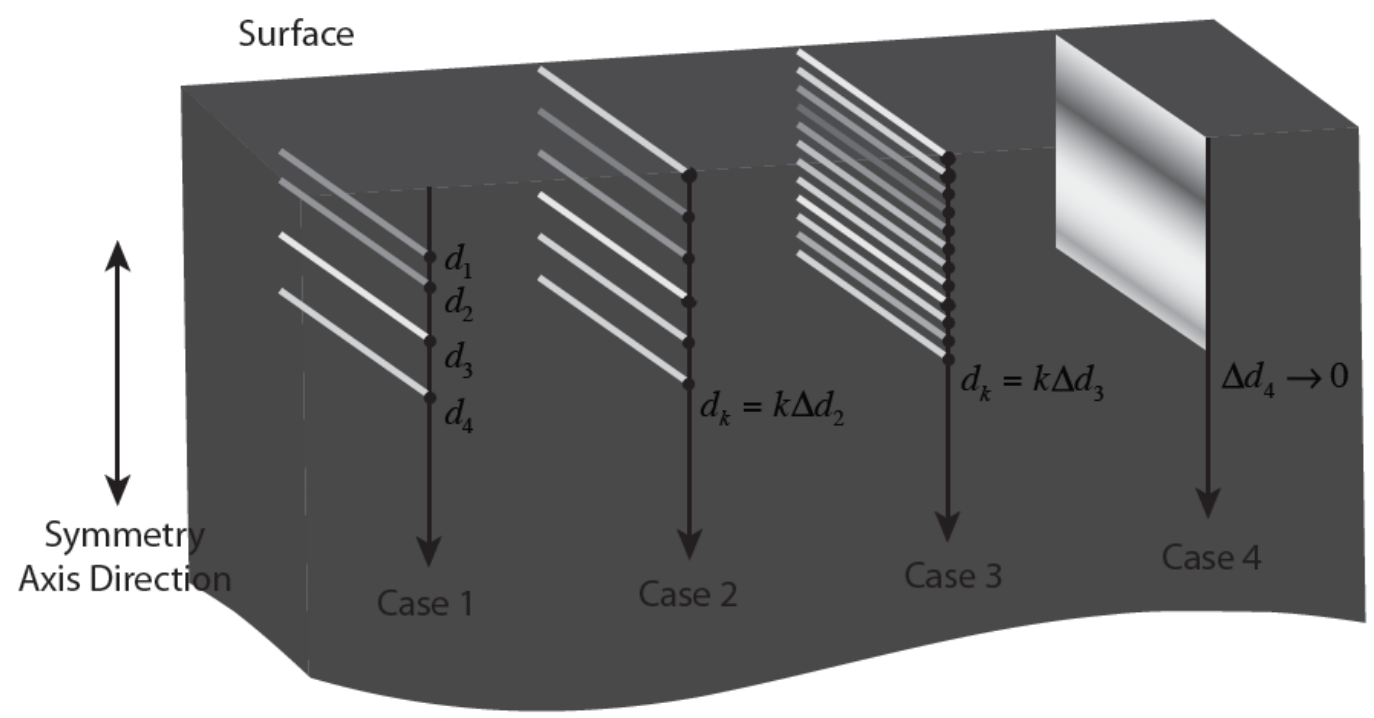

